Published in final edited form as:

Nat Mater. 2015 September ; 14(9): 951-960. doi:10.1038/nmat4350.

\title{
Fractal heterogeneity in minimal matrix models of scars modulates stiff-niche stem-cell responses via the nuclear exit of a mechanorepressor
}

\author{
P. C. Dave P. Dingal ${ }^{1, \neq}$, Andrew M. Bradshaw ${ }^{1}$, Sangkyun Cho ${ }^{1}$, Matthew Raab ${ }^{1}$, Amnon \\ Buxboim ${ }^{1}$, Joe Swift ${ }^{1}$, and Dennis E. Discher ${ }^{1, *}$ \\ ${ }^{1}$ Biophysical Engineering Labs for Molecular \& Cell Biophysics and NanoBio-Polymers, \\ Department of Chemical and Biomolecular Engineering, University of Pennsylvania, Philadelphia, \\ Pennsylvania 19104, USA
}

\begin{abstract}
Scarring is a long-lasting problem in higher animals, and reductionist approaches could aid in developing treatments. Here, we show that co-polymerization of collagen-I with polyacrylamide produces minimal matrix models of scars (MMMS), in which fractal-fiber bundles segregate heterogeneously to the hydrogel subsurface. Matrix stiffens locally - as in scars - while allowing separate control over adhesive-ligand density. The MMMS elicits scar-like phenotypes from mesenchymal stem cells (MSCs): cells spread and polarize quickly, increasing nucleoskeletal lamin-A yet expressing the 'scar marker', smooth muscle actin (SMA) more slowly. Surprisingly, expression responses to MMMS exhibit less cell-to-cell noise than homogeneously stiff gels. Such differences from bulk-average responses arise because a strong SMA repressor, NKX2.5, slowly exits the nucleus on rigid matrices. NKX2.5 overexpression overrides rigid phenotypes, inhibiting SMA and cell spreading, while cytoplasm-localized NKX2.5 mutants degrade in well-spread cells. MSCs thus form a 'mechanical memory' of rigidity by progressively suppressing NKX2.5, thereby elevating SMA in a scar-like state.
\end{abstract}

\section{Keywords}

Matrix; Polymer Segregation; Mechanobiology; Mechanorepressor

\begin{abstract}
Scars are a lasting consequence of injuries that range from acute events such as myocardial infarctions and traumatic insults to numerous chronic diseases ${ }^{1}$. A defining feature of a scar is a fibrotic, heterogeneous, and stiffened matrix across tissues as diverse as $\operatorname{liver}^{2}$, lung $^{3}$, and striated muscle $\mathrm{e}^{4,5}$ (Fig. 1a,b). Tissue culture plastic and glass coverslips are also stiff, but neither of these homogeneous ubiquitous materials has the heterogeneous microarchitecture of a scar, and the innumerable labs that study cells on plastic, glass, or even a homogeneous gel are unlikely to consider that their cells have phenotypes as if grown on/in
\end{abstract}

\footnotetext{
Users may view, print, copy, and download text and data-mine the content in such documents, for the purposes of academic research, subject always to the full Conditions of use:http://www.nature.com/authors/editorial_policies/license.html\#terms

*discher@seas.upenn.edu.

†Present address: Department of Bioengineering, Stanford University, Stanford, California 94305, USA.
} 
scar tissue. On very soft gels, a cell's average shape and cytoskeleton differ considerably from those on rigid substrates ${ }^{6}$, but it is unknown whether a soft tissue-like gel containing a minor volume fraction of scar-like fibers could elicit cell responses even partially equivalent to any homogeneous substrate. In addition, whether materials influence variations in responses (eg. gene expression noise) of cell populations can also be important for understanding and for using cells in therapy, especially stem cells that proliferate and differentiate in response to materials. We sought therefore to develop heterogeneous, scarlike gel systems in order to compare phenotypes and their cell-to-cell variations to homogeneous materials of different stiffness.

Collagen-I is the most abundant protein in mammals, but the partially oriented and de novo bundles of crosslinked collagen-I in a scar have been characterized as having an atypical fractal ${ }^{7}$ micro-architecture, the way tree branches fill space. The fiber bundles displace normal tissue and thereby limit tissue function ${ }^{8}$. In the scarring that occurs in muscle diseases for example, collagen-I ( $C O L 1$ ) expression increases up to ten-fold or more relative to normal levels together with many additional changes in scarred tissue ${ }^{9}$ (Fig. 1c,

Supplementary Fig. S1). Upregulation of the ACTA2 gene, which produces the 'scar marker' smooth muscle actin (SMA), indicates increased cell tension ${ }^{10}$, and it is expressed many days after injury in spindle-shaped cells, remaining high in scars for a decade or more ${ }^{11}$. Upregulation of the nuclear structure protein lamin-A ( $L M N A)$, together with the retinoic acid-modulated transcription factor $R A R G$ that regulates $L M N A$ levels, is consistent with recent correlations between lamin-A and collagen-I levels in normal tissues ${ }^{12}$ - but kinetics are unclear for this apparent relationship. Large decreases in expression of at least one gene that encodes for a "heart development" transcription factor, $N K X 2-5$, are also evident in diseased skeletal muscle (Fig. 1c), which hints at a much broader role than previously considered ${ }^{13}$ for such a regulatory factor. The complexity of cell types, matrix, and soluble factors in scars in vivo confounds whether any particular cell type responds per such profiles to the fractal heterogeneity of a scar microenvironment. Our reductionist goal here was to develop a controllable minimal matrix model for 2D cultures that possesses a microarchitecture with fractal heterogeneity and inherently variable stiffening observed in scars and that also causes a relevant cell type to respond as if in a 3D scar.

For many types of injured and scarred tissues, various endogenous cell types including mesenchymal stem cells (MSCs) might impact the collagen at the injured site, but therapies are certainly being pursued with MSCs ${ }^{14,15}$. MSCs in vitro are not only multipotent ${ }^{14}$, but also mechanosensitive ${ }^{16}$. Whether these cells or derived lineages are 'plastic' in changing phenotype when engrafted into a scar - or even when expanded in culture before injection is a critical issue for cell therapy. MSCs that are purified from soft marrow $(\sim 300 \mathrm{~Pa})^{17}$ and cultured conventionally on rigid plastic ${ }^{18}$, or that are derived from embryonic stem cells ${ }^{19}$ exhibit expression profiles that are similar in some key ways to those of injured tissues; particularly, the progressive increases in $L M N A$ and ACTA2, which anti-correlate with NKX2-5 (Fig. 1c). These transcript profiles provide hints of expression changes of possible relevance to scars and motivate the detailed studies here of protein dynamics and mechanisms in MSCs as a suitable cell type on substrates with scar-like features rather than homogeneously rigid plastic. 
Rigid-on-soft composite substrates have recently been made with micropatterns ${ }^{20}$, although effects of local elastic modulus were not decoupled from ligand density. Matrix ligand type and density provide signals complementary to those possible from matrix mechanics in influencing cell behavior such as the differentiation of $\mathrm{MSCs}^{21-23}$. However, recent studies in culture of matrix tethering ${ }^{24}$, topography ${ }^{25}$, crosslinking and growth in $3 \mathrm{D}^{22,23}$ have also stirred debate on the influence of matrix properties in cell-fate decisions. Indeed, the stiffness $(E)$ of tissues including scars when measured on a macroscopic scale sometimes lead to a far greater stiffness $(\mathrm{MPa})^{26}$ than when measured on the microscopic scale $(\mathrm{kPa})^{27}$. Expression profiles of cells on culture substrates of various properties can be compared with tissue profiles (e.g. Fig. 1c) in order to clarify relationships to specific tissue parameters - an important goal of new materials systems for cell culture ${ }^{28}$. Here, with a minimal matrix model of scars in culture, we make a range of comparisons to scarred tissue (Fig. 1a-c and Supplementary Fig. S1) and ultimately provide evidence that the effective stiffness of a matrix with fractal heterogeneity like a scar is key to cell-fate signals and mechanical memory of the cell.

\section{Heterogeneously Rigid Minimal Matrix Models of Scars (MMMS)}

Starting with an allylsilane-modified 'bottom' coverslip that anchors polyacrylamide (PA) chains, we discovered that mixing collagen-I $(0.04 \% \mathrm{w} / \mathrm{v})$ heterotrimers into the PA precursor solution promotes collagen fiber formation that is laterally heterogeneous (Fig. 1d) and also segregated to within $\sim 10 \mu \mathrm{m}$ of the interface of an $\sim 80 \mu \mathrm{m}$ thick gel during freeradical polymerization (Supplementary Fig. S2). Collagen concentration thus increases into the lower range of that in scarred muscle tissue (see Supplementary Discussion), and collagen also formed oriented fibrous regions with a mean fractal dimension similar to that reported for scars ${ }^{7}$ as opposed to tissue culture plastic (Supplementary Fig. S2). Cells added to these embedded-collagen gels do not adhere or spread, consistent with sub-surface fibers with inaccessible adhesive ligand (Supplementary Fig. S3). Further modification of the surface of the PA gel (nominal $E=0.3 \mathrm{kPa}$, unless specified otherwise) with collagen-1 coating (CC) via a standard sulfo-SANPAH-mediated covalent reaction ${ }^{29}$ produces a celladhesive minimal matrix model of a scar (MMMS; Fig. 1d and Supplementary Fig. S4). The fractal fiber bundles of purely structural collagen prove stable for at least 7 days in cell culture (Supplementary Fig. S3). The minimal dimensionality of the 2D culture model is shown below to be sufficient to cause cell expression changes consistent in trends with 3D fibrotic tissue (Fig. 1c). The MMMS thus provides decoupled control over heterogeneous matrix physical properties and ligand density, which is not possible in pure collagen gels where ligand density varies with collagen density and can change with proteolysis. Furthermore, unlike PA gels that are linearly elastic, collagen gels are non-linear ${ }^{30}$ and stiffen under strain generated by incorporated cells ${ }^{31}$, complicating interpretations of matrixmechanical effects on cells.

Consistent with the sub-surface formation of fiber bundles, the gel's bulk modulus was controlled by varying the ratio of acrylamide to bis crosslinker (for $E=0.3-40 \mathrm{kPa}$ ) independent of collagen, and for all gels, the elastic modulus dominated the viscous modulus (Supplementary Fig. S2). However, because a surface-adherent cell feels only a few microns deep into a gel ${ }^{32}$, the surface microelasticity $E_{\text {surf }}$ seemed critical to measure. We started 
with conventional micro-indentation by atomic force microscopy (AFM $)^{33}$, with results for pristine or CC gels identical to bulk rheology (Supplementary Fig. S4). Microelasticity maps of MMMS gel surfaces proved to be far more heterogeneous than those of CC gels, and the MMMS gels also appeared 2-3-fold stiffer than the same gels without collagen fibers (Supplementary Fig. S4). However, such a nominal shift for a stratified material is indicative of a stiffer material on top of a softer bulk. We calculated therefore a suitable thin-film correction and also measured gel tackiness for a second estimation of $E_{\text {surf }}$ (see Supplementary Discussion). Both measurements suggested $E_{\text {surf }} \sim 10 \mathrm{kPa}$, which is far stiffer than the bulk gel.

Cells apply tractions predominantly along the matrix surface ${ }^{29}$, whereas the AFM probes primarily normal to the substrate. Lateral pulling of the gel surface with a glass microprobe at constant displacement was therefore used to apply a constant force on MMMS gels. Displacements of microbeads embedded in the gels exhibited the expected Boussinesq-like profiles (Supplementary Fig. S4). Displacement profiles far from a fiber bundle were strikingly similar to those of homogeneous $0.3 \mathrm{kPa}$ gels, whereas profiles closer to fiber bundles appeared similar to those of homogeneous $10 \mathrm{kPa}$ gels (Fig. 1e). We estimated therefore that $E_{\text {surf }} \sim 0.3$ to $10 \mathrm{kPa}$ of MMMS, which highlights the near-field rigidity of the fiber bundles. The concentration of embedded collagen-1 was chosen to approximate the Sirius Red area fraction visible in typical sections of scarred tissue $(\sim 20-30 \%)^{34}$. Use of Sirius Red here confirms what immunofluorescence (Fig. 1d) already shows as collagen fiber bundles in MMMS but not in CC gels (Fig. 1f) and is consistent with staining of a thin section of fibrotic liver (Fig. 1g). The imaging and physical measurements thus reveal the MMMS substrates as a stiff fibrous meshwork within a background of an otherwise soft matrix.

\section{Cell-averaged MMMS Rigidity induces Early \& Late Responses}

Whether cells respond in a scar-like manner was the next critical question. Immunostaining and imaging of MSCs on soft gels $\left(\mathrm{CC}_{0.3 \mathrm{kPa}}\right)$ versus MMMS confirmed the expected myosin-IIB (MIIB) polarization toward the cell rear ${ }^{35}$ as well as differences in expression of smooth muscle actin (SMA) (Fig. 2A). SMA is a standard marker for intracellular tension and is upregulated in various cell types in scarred tissue ${ }^{10}$ (Fig. 1c).

Our various measures of cell morphology and cytoskeletal organization/level for MSCs on MMMS are indistinguishable from MSC responses on homogeneous CC gels of 10-14 $\mathrm{kPa}$. Most cells spread more on stiffer substrates ${ }^{16,36}$, leading to larger projected areas of cells and nuclei (Fig. 2b), as fitted by hyperbolic responses to matrix $E$ predictable from contractile dipole models ${ }^{33,36}$. From the data and fit for homogeneous gels, the MMMS gel possesses an average effective elasticity $\left(E_{\text {eff }}\right.$ ) of $\sim 12 \mathrm{kPa}$ despite matrix heterogeneity. Aspect ratios of cells on MMMS gels are near the peak aspect ratio for cells on homogeneous gels of this same $\sim 12 \mathrm{kPa}$ stiffness (Supplementary Fig. S5), which demonstrates that - unlike stiff substrates and rigid plastic - matrices of intermediate stiffness maximize the spindle-shape phenotype. However, stiffness-dependent spreading is characteristic of low-passage MSCs since prolonged culture of primary MSCs on rigid plastic (to high passage numbers) increasingly promotes substrate-independent spreading 
and cell tension (with more SMA-positive stress fibers) (Fig. 2c). Thus, for low-passage MSCs, MIIB polarization at day-1 and day-2 preceded SMA upregulation on MMMS (Fig. $2 \mathrm{~d}$, left and right); myosin-dependent durotactic migration on hybrid CC0.3kPa/MMMS gels (Supplementary Figs. S5, S6) and in 3D heart-derived, graded, crosslinked matrices (Supplementary Fig. S7) further confirmed the role of nonmuscle myosins in the early, dynamic, matrix-stiffness response. The mechanosensitive nucleoskeletal protein, lamin$\mathrm{A}^{12}$, increased within 1 day of substrate adhesion (Fig. 2d, middle), concomitant with reduction of phosphorylation (at serine-22, which solubilizes lamin-A for turnover ${ }^{12}$; Fig. $2 \mathrm{~d}$, middle inset). Nucleus shapes also respond similarly to matrix rigidity-induced contraction (Supplementary Fig. S5). SMA upregulation was only significant after day 2, and its expression was hyperbolic with $E\left(R^{2} \geq 0.97\right)$, and once again indicated that the MMMS has an $E_{\text {eff }} \sim 12 \mathrm{kPa}$ (Fig. 2d, right inset). Thus, although the 'scar marker' SMA eventually correlates with early-responding lamin-A and is indirectly down-regulated by retinoic acid that directly controls lamin-A (Supplementary Fig. S8), SMA induction is not required for early stiffness sensing. Liver fibrosis caused by chemical toxin likewise shows SMA is a late marker of scarring ${ }^{2}$.

\section{Expression Noise on MMMS differs from Homogeneous Gels}

Immunofluorescence imaging confirmed SMA upregulation and its incorporation into stress fibers in many MSCs on MMMS and $\mathrm{CC}_{40 \mathrm{kPa}}$ gels relative to $\mathrm{CC}_{0.3 \mathrm{kPa}}$ gels, and immunoblotting confirmed these overall increases in SMA and also caldesmon (Fig. 3a,b). However, imaging revealed considerable expression noise between cells, especially on the $\mathrm{CC}_{40 \mathrm{kPa}}$ gels. To facilitate comparisons, all experiments included cultures on $\mathrm{CC}_{0.3 \mathrm{kPa}}$ gels so that the mean level of a protein on the soft gels could be used to normalize results from stiffer substrates in the same experiment. A substantial fraction (50 60\%) of cells on any of these stiffer substrates possesses low levels of both SMA and the polarization marker MIIB, similar to the low levels in cells on soft $\mathrm{CC}_{0.3 \mathrm{kPa}}$ matrix (Fig. $3 \mathrm{c}-\mathrm{d}$ and Supplementary Fig. S8). On soft gels, MSCs lack SMA stress fibers (Fig. 3a) and are a single-peak population (Fig. 3c). MMMS has both soft regions and stiffer, fractal fiber islands, and so it is natural to identify the low-SMA expressing cells as "Non-responders" that are - in principle influenced more by the soft regions than the stiffer islands. The same subpopulation also shows low MIIB (Supplementary Fig. S8). For all substrates, we therefore subtract out this Non-responder peak from the entire population (after first re-scaling to the grey distribution) in order to obtain the subpopulations of Responders with high SMA and high MIIB (e.g. Fig. 3c, right histogram and Supplementary Fig. S8).

In quantifying single cell expression cells on MMMS seem to show less variance than cells on gels of homogeneous stiffness. The long tail of high-SMA expressors that is evident for Responders on $\mathrm{CC}_{40 \mathrm{kPa}}$ gels (Fig. 3c) is also apparent for these stiff gels and for $\mathrm{CC}_{10 \mathrm{kPa}}$ gels in box-and-whisker scatterplots of the entire populations: both the box surrounding $70 \%$ of the data and the whiskers bracketing $90 \%$ of the data are larger for stiff gels than for MMMS (Fig. 3d). High-throughput flow-cytometry analysis of trypsin-detached and immunostained cells ${ }^{37}$ supports the trends in cell-cell heterogeneity of SMA and also of myosin-II activity levels (Supplementary Fig. S8). Noisy expression of single cells in standard culture dishes has been quantified in terms of cell-to-cell standard deviation/mean 
$(\sigma / \mu)$, with one recent study of micro-RNA showing noise in its target protein varying from $(\sigma / \mu)=0$ to 1 depending on the micro-RNA ${ }^{38}$. Such studies of expression noise have yet to describe matrix effects, but we find for soft matrix that $(\sigma / \mu)_{\mathrm{CC} 0.3 \mathrm{kPa}} \approx 0.5 \pm 0.07$ for SMA (Fig. 3c) and that stiffer matrices might increase this up to 5-fold (Fig. 3e). However, MMMS consistently suggests lower expression noise than homogeneous $\mathrm{CC}_{10 \mathrm{kPa}}$ or $\mathrm{CC}_{40 \mathrm{kPa}}$ matrices in analyses of entire populations and of responder subpopulations (Fig. 3e, right plot).

A Kolmogorov-Smirnov test (KS test) of two populations provides a second, nonparametric analysis sensitive to complicated shapes of distributions. KS tests of single cell expression profiles by Rinkevich et $\mathrm{al}^{39}$ used similar numbers of cells and criteria as here to identify fibroblastic cell types that contribute to scarring. Based on KS tests for SMA and MIIB at day-2 and day-7, the entire populations of cells on MMMS and $\mathrm{CC}_{40 \mathrm{kPa}}$ matrices differ from cells on $\mathrm{CC}_{0.3 \mathrm{kPa}}$ (Fig. 3f). Responder cell distributions on MMMS also differ from those on $\mathrm{CC}_{10 \mathrm{kPa}}$ and $\mathrm{CC}_{40 \mathrm{kPa}}$ (Fig. 3f), and because cells on MMMS exhibited noise levels closest to $(\sigma / \mu)_{\mathrm{CC} 0.3 \mathrm{kPa}}$ (Fig. 3e), the KS test was further applied to distribution shapes for Responders versus Non-responders (Fig. 3f, bottom Table). For MMMS, the two populations appear statistically similar in SMA variance ( $p=0.19$, Fig. 3f; compare Fig. 3c, left: grey disribution to Fig. 3c, right: MMMS Responder distribution), suggesting that the entire population on MMMS is bimodal, whereas cell subpopulations on homogeneously stiff substrates are not bimodal $\left(\mathrm{CC}_{0.3 \mathrm{kPa}}\right.$ vs $\mathrm{CC}_{10 \mathrm{kPa}}, p=6 \times 10^{-13} ; \mathrm{CC}_{0.3 \mathrm{kPa}}$ vs $\mathrm{CC}_{40 \mathrm{kPa}}, p=$ $\left.3 \times 10^{-6}\right)$. This is consistent with the many more distinctly intense cells on homogeneously stiff substrates (Fig. 3a and Supplementary Fig. S9). Importantly, cell-scale measurements of scarred muscle (Fig. 1b) appear bimodal with soft and stiff modes that are similar ( $p=$ 0.997) and AFM measurements of MMMS microelasticity also appear bimodal (Supplementary Fig. S4). Cell tension - which SMA reveals - contributes significantly to injured tissue stiffness ${ }^{2}$, and likely explains the similarity between bimodal SMA expression in cells on MMMS matrices and bimodal stiffening in vivo. Surprisingly, homogeneous stiff substrates might cause even greater cell-cell heterogeneity than MMMS. Given the importance of niche-sensitivity of stem cells, a bio-inspired matrix with fractal heterogeneity might thus present a materials advantage over homogeneously stiff surfaces in terms of noise-suppression.

While stiffness-dependent increases in SMA are consistent with fibrotic tissue (Fig. 1c) and a progressive commitment to a scar-like phenotype ${ }^{11}$, the expected decreases of NKX2.5 in fibrotic tissue (Fig. 1c) were not evident in 7-day cultures of MSCs on MMMS or $\mathrm{CC}_{40 \mathrm{kPa}}$, compared to $\mathrm{CC}_{0.3 \mathrm{kPa}}$ gels (Fig. 3b). Changes in subcellular localization of NKX2.5 seemed a possible determinant in mechanism as did longer times in culture.

\section{Slow Nuclear Loss of a Mechanorepressor on Rigid Matrix}

Transcription factors including RARG ${ }^{12}$, serum response factor $(\mathrm{SRF})^{40}$, and YAP/TAZ ${ }^{41}$ enter the nucleus of multiple cell types on stiff substrates and promote expression of some mechanosensitive genes. NKX2.5 (capitalized, if human) is often described as a transcription factor critical to cardiogenic specification ${ }^{13}$, but below we show in human bone marrow-derived MSCs that NKX2.5 is a mechanosensitive repressor of SMA. Of the 
many $N K X$ isoforms in microarray profiles of MSCs, $N K X 2-5$ is also the most abundant (Supplementary Fig. S10).

To determine whether NKX2.5 can play a role in a cell's mechano-response to matrix, embryonic cardiomyocytes beating on soft or stiff gels were fixed and immunostained, revealing Nkx2.5 to be mostly nuclear but with higher total Nkx2.5 on stiff-gel cultures because of increased cytoplasmic levels (Supplementary Fig. S10). Recent evidence of NKX2.5 in MSCs focused on cardiogenic differentiation when cultured on extracellular matrix derived from healthy or infarcted hearts and attached to stiff gels ${ }^{42}$, but cardiogenesis of MSCs seems unlikely ${ }^{43}$. In MSCs on soft gels, NKX2.5 is nuclear-localized, but unlike cardiomyocytes, it is predominantly cytoplasmic on MMMS and stiff gels (Fig. 4a and Supplementary Fig. S9). Nuclear exit of NKX2.5 in MSCs occurred over days, which is much slower than SRF mechano-activation that can respond within minutes ${ }^{40}$ (Fig. $4 \mathrm{~b}$ ), suggesting that SRF drives initial expression of SMA. Transcript profiling after $\sim 1.5$ days (Fig. 4b, left inset) revealed stiffness-driven increases in ACTA2 and CALD1 but not $N K X 2-5$ or $S R F$ (which promotes its own expression), consistent with the more important role of localization rather than abundance. Importantly, even within a given culture, variations in SMA versus nuclear NKX2.5 (Fig. 4b and Supplementary Fig. S9) fit well to a cooperative inhibition model (see Fig. $4 \mathrm{~b}$ legend), and a cooperativity exponent 2.7 indicates a switch-like transition between at least two cell phenotypes: (NKX2.5-high, SMA-low) and (NKX2.5-low, SMA-high).

Since a fraction of cytoplasmic NKX2.5 localized to SMA-rich stress fibers in cells on stiff matrix (Fig. 4c), stress fibers were disrupted with blebbistatin that inhibits myosin-II's ATPase. Within hours, nuclear NKX2.5 levels increased and SMA levels decreased (Fig. 4d). Transcript profiles following blebbistatin treatment of MSCs on both stiff and rigid substrates $\left(\mathrm{CC}_{10 \mathrm{kPa}}\right.$ and rigid plastic) further showed the expected repression of ACTA2, but in the absence of reproducible changes in cardiac-related transcripts downstream of NKX2.5 (Fig. 4e). As a nuclear mechanosensor, lamin-A decreased as expected ${ }^{12,41}$ together with target genes of YAP $1^{49}$ and SRF (slightly), with the latter likely to also contribute to the $>50 \%$ decrease in SMA at both protein and transcript levels.

To determine possible regulatory factors for NKX2.5 localization, whole cell lysates were made from rigid-substrate cultures, immunoprecipitated (IP) with anti-NKX2.5, and analyzed by mass spectrometry (MS). Of 31 proteins specifically pulled down (versus nonspecific $\mathrm{IgG}), 90 \%$ have gene ontology terms associated with the cytoplasm and only 3 are strictly nuclear (Fig. 4f). Specificity of the anti-Nkx2.5 was validated with Nkx2.5overexpressing A549 cells that show strong nuclear localization (Fig. $4 \mathrm{f}$ image) but with little to no endogenous NKX2.5, and the IP-MS analysis yielded seven unique Nkx2.5 peptides (Supplementary Fig. S10). Moreover, IP-MS of overexpressing cells shows more nuclear interaction partners (Supplementary Table S3). Immunofluorescence confirmed that overexpressed NKX2.5 localized to the nucleus (of late-passage MSCs on rigid substrates) and also decreased cell area and SMA levels (Fig. 5a).

To further clarify the basis for matrix rigidity-dependent translocation of NKX2.5 between nucleus and cytoplasm, mutants of the nuclear localization signal (NLS) in Nkx2.54 were 
transiently transfected into MSCs cultured on rigid substrates. Wild-type, NLS1, and NLS2 mutants were nucleus-enriched whereas NLS3 was predominantly cytoplasmic (Fig. 5b). Nuclear-localized wild-type, NLS1, and NLS2 mutants repressed SMA, reduced stress fibers, and ultimately reduced cell and nuclear spread areas on rigid substrate (Fig. 5c,d). Cytoplasmic-localized NLS3 differs by as few as 3 amino acids from NLS1 and NLS2 and not only maintained the rigid-substrate phenotype but also showed the lowest total Nkx2.5 level (despite being studied at the same time; Fig. 5c, inset). Cytoplasmic Nkx2.5 was also found in lamellipodia where myosin phosphatase-1 (MYPT1) is co-localized (Supplementary Fig. S10), consistent with MYPT1-dependent nuclear exit of NKX2.5 in embryonic stem cells $\mathrm{s}^{45}$.

Conventional cultures of primary MSCs that are passaged for weeks on rigid plastic exhibit a 'mechanical memory' 46 with more cells that are large and SMA+ when re-plated on a soft matrix (Fig. 2c). Consistent with this, we find a large and progressive decrease in total NKX2.5 (Fig. 6a) that appears in immunoblots as the expected doublet at 56-58 $\mathrm{kDa}^{45,47}$, as validated by MS (Supplementary Fig. S10 and Table S3). Rigidity-driven osteogenesis ${ }^{16}$ can also occur - relevant perhaps to ectopic calcification ${ }^{48}$, but while MMMS is similarly osteo-inductive and suppressible by retinoic acid, calcification is limited on MMMS like that on soft matrix (Supplementary Fig. S11). MMMS matrices with their minor volume fraction of stiff fractal fibers thus differ in several key ways from homogeneous gels, rigid plastic dishes, and coverslips in terms of cell phenotype.

\section{Outlook}

Tissue models of fibrosis can be generated in various ways such as by acute soluble factorinduced injury (e.g. carbon tetrachloride ${ }^{2}$ ) but are complicated by many factors, including immune responses, changes in density of adhesive ligands, matrix heterogeneity, and timescales for fibrosis of weeks - years. A separation of time scales is indeed evident for MSCs on MMMS, with some molecules responding in hours while the key mechanorepressor NKX2.5 only slowly exits the nucleus and degrades to build an SMA+ 'mechanical memory' of rigidity (Fig. 6b). Additional questions for reductionist models include how far away does a cell feel a scar, can other cell types exhibit expression changes in fibrotic tissue (e.g. Fig. 1b,c), and does cancer-associated fibrosis (e.g. Fig. 1g) affect cell-cell variations in cancer stem cells? Mechanobiological gene circuits ${ }^{49}$ might eventually predict answers to such questions (see Supplementary Discussion), and particularly important will be spatiotemporal models for MMMS which incorporate strong repression by NKX2.5 (Fig. 4b) in order to show that when an SMA-high cell (strong Responder) on a fractal fiber island migrates onto soft matrix it becomes a weak Responder in a way that cannot happen on homogeneous substrates (Supplementary Fig. S9). Observing such dynamics within in vivo scars will be challenging, but MMMS can point in other useful directions. Indeed, single cell profiling of fibroblasts recently implicated a key role in scarring for the drug-inhibitable peptidase Cd26/Dpp $4^{39}$, consistent with $C D 26$ in MSCs decreasing with NKX2.5 overexpression and with LMNA knockdown (Supplementary Fig. S12). Beyond CD26, NKX2.5, and retinoids that regulate $L M N A$ and indirectly SMA (Supplementary Fig. S8,S11), MMMS-type materials might help advance other drugs, pathways, and stem cell types into needed treatments of fibrosis. 


\section{Methods}

\section{Synthesis and functionalization of conventional and MMMS hydrogels for cell culture}

Circular glass coverslips (18-mm diameter, Fisher Scientific) were pre-treated in boiling ethanol, then RCA solution at $70^{\circ} \mathrm{C}\left(1: 1: 3\right.$ of $30 \% \mathrm{H}_{2} \mathrm{O}_{2}: 15 \mathrm{~N} \mathrm{NH}_{4} \mathrm{OH}$ :distilled $\left.\mathrm{H}_{2} \mathrm{O}\right)$ for 10 minutes each, and functionalized in chloroform solution with $0.1 \%$ allyltrichlorosilane and $0.1 \%$ triethylamine for half an hour. Varying ratios of N,N'-methylene-bis-acrylamide (Sigma) and acrylamide solution (40\%, Sigma) in distilled $\mathrm{H}_{2} \mathrm{O}$ were formulated to tune polyacrylamide (PA) hydrogel stiffness. The PA precursor solution $(20 \mu \mathrm{L})$ was pipetted on allylsilanated coverslips with $0.1 \%$ ammonium persulfate and $0.1 \% \mathrm{~N}, \mathrm{~N}, \mathrm{~N}^{\prime}, \mathrm{N}^{\prime}-$ tetramethylethylenediamine to initiate polymerization. To make a $0.3 \mathrm{kPa} P A$ gel, precursor solution consists of $3 \%$ acrylamide and $0.07 \%$ bis-acrylamide; a $40 \mathrm{kPa}$ PA gel comprises $10 \%$ acrylamide and $0.3 \%$ bis-acrylamide (Supplementary Table S1). To create PA gels with embedded collagen (e.g. MMMS), type I rat-tail collagen (BD Biosciences) was diluted in the $0.3 \mathrm{kPa}$ PA precursor solution to a final concentration of $0.4 \mathrm{mg} / \mathrm{mL}$ in $50 \mathrm{mM}$ HEPES buffer (pH 8). To functionalize the polymerized PA gel, $300 \mathrm{uL}$ of sulfo-SANPAH (Thermo Scientific, $0.5 \mathrm{mg} / \mathrm{mL}$ in HEPES), was added to the gel and exposed for 7 min under $365-\mathrm{nm}$ UV light (0.7 A, Spectroline Model XX-15A), and was washed twice in distilled $\mathrm{H}_{2} \mathrm{O}$. Collagen-I was diluted to $0.1 \mathrm{mg} / \mathrm{mL}$ in HEPES and incubated with the functionalized PA gel at $37^{\circ} \mathrm{C}$ overnight on a shaker. Excess collagen was removed and the gel was equilibrated in PBS. Cells were plated onto the gels within 24 hours of collagen attachment. Preliminary studies on the efficacy of sulfo-SANPAH as a collagen-PA gel crosslinker reveal that the collagen- 1 concentration $(0.1 \mathrm{mg} / \mathrm{mL})$ used in all experiments here, as suggested by previous studies ${ }^{33,52}$, saturates all available reactive moieties on the PA gel and are covalently tethered to the PA gel (Supplementary Fig. S4). These studies altogether indicate saturation of matrix ligands for cells, thus eliminating the possibility of cell rounding due to low ligand density.

\section{Surface characterization and microrheology of hydrogels}

Sirius Red dye ( $0.1 \%$ in $\mathrm{H}_{2} \mathrm{O}$, Direct Red 80 , Sigma) was stained (for $1 \mathrm{~h}$, rinsed twice in $0.5 \%$ acetic acid) on embedded collagen fibers in the gel to determine their scar-matrix mimesis. Elasticity of the gel surface $E_{\text {surf }}$ was quantified with an Asylum MFP-3D Atomic Force Microscope (Asylum Research, Santa Barbara), equipped with a microscope stage micromanipulator to move across the gel during AFM probing. The spatial AFM resolution used here is $1 \mu \mathrm{m}$, which emulates cellular length scales in matrix sensing. A silicon nitride cantilever probe with pyramidal tip and spring constant of 30-100 pN/nm (MCST, Veeco) was used to indent the gels. To determine $E_{\text {surf }}$, the Hertz cone model was used to fit probe deflection curves (up to $1 \mu \mathrm{m}$ indentation) ${ }^{53}$. In lateral pulling experiments (Fig. 1d), glass micropipettes were formed from glass capillaries (1-mm inner diameter, World Precision Instruments, Sarasota, FL) using a Flaming-Brown Micropipette Puller (Sutter Instrument, Novato, CA). Pulled micropipettes were tapered at the base, were reopened to a final inner diameter of $10 \mathrm{um}$, and were used to probe the surface of gels, embedded with microbeads (1- $\mu \mathrm{m}$ diameter FluoSpheres, Invitrogen) as fiduciary markers. Glass probe was constantly displaced $20 \mu \mathrm{m}$ parallel to the gel surface, and fluorescent bead displacements were acquired with a Nikon Eclipse TE300 inverted microscope equipped with Cascade 
Photometric CCD camera. Particle Image Velocimetry, an ImageJ plugin, was used to quantify fiduciary bead displacements after probe pulls. Analysis of displacement profiles is detailed in Supplementary Information.

\section{Analysis of cell-to-cell expression noise}

The effect of matrix mechanics and heterogeneity on single-cell SMA and MIIB responses in day- 2 and day- 7 cultures on various matrices were compared using noise levels (ratio of standard deviation to mean, $\sigma / \mu$ ) and higher-order expression modes (two-sample Kolmogorov-Smirnov test). The former metric is the same as in various studies of stochastic gene expression ${ }^{38}$ but used here to for matrix effects on response noise. The 'KS test' is the same as in recent studies of scar-inducing fibroblasts ${ }^{39}$ and is nonparametric and more sensitive to both shape and location of distributions. To specifically compare responding populations on different substrate, the Non-responder modes (e.g. all cells on $\mathrm{CC}_{0.3 \mathrm{kPa}}$ ) were proportionally removed from response histograms of a given substrate condition, based on the maximum frequency of the Non-responder peak.

\section{Cell culture and treatments}

Human MSCs, isolated from human donor bone marrows (anonymous donors with Institutional Review Board approvals) by sorting CD34-negative cells, were obtained from Stem Cell and Xenograft Core, University of Pennsylvania School of Medicine, and cultured using standard methods ${ }^{14}$. Cells were expanded in tissue culture flasks (Corning, Inc.) and used at passages 3-5 (unless otherwise noted) for all cell-on-gel cultures in low-glucose DMEM supplemented with $10 \%$ FBS and $100 \mu \mathrm{g} / \mathrm{ml}$ penicillin and $100 \mu \mathrm{M}$ streptomycin. Racemic blebbistatin (EMD Biosciences) was used at $50 \mu \mathrm{M}$.

Plasmids that encode for mouse Nkx2.5 with mutated nuclear localization sequences (NLS; residues 135-141) were graciously provided by Dr. Kasahara (University of Florida). The putative NLS sequence is RRRRKPR, which was mutated to RRRRAAA (NLS1), AAAAKPR (NLS2) or AAAAAAA (NLS3) ${ }^{44}$. A549 cells were transfected with wild-type Nkx2.5 and were harvested to validate the NKX2.5 antibody by mass spectrometry (Supplementary Fig. S10; see Supplementary Methods).

\section{Supplementary Material}

Refer to Web version on PubMed Central for supplementary material.

\section{References}

1. Gurtner GC, Werner S, Barrandon Y, Longaker MT. Wound repair and regeneration. Nature. 2008; 453:314-321. [PubMed: 18480812]

2. Georges PC, et al. Increased stiffness of the rat liver precedes matrix deposition: implications for fibrosis. American journal of physiology. Gastrointestinal and liver physiology. 2007; 293:G1147G1154. [PubMed: 17932231]

3. Liu F, et al. Feedback amplification of fibrosis through matrix stiffening and COX-2 suppression. J Cell Biol. 2010; 190:693-706. [PubMed: 20733059] 
4. Berry MF, et al. Mesenchymal stem cell injection after myocardial infarction improves myocardial compliance. American journal of physiology. Heart and circulatory physiology. 2006; 290:H2196H2203. [PubMed: 16473959]

5. Engler AJ, Rehfeldt F, Sen S, Discher DE. Microtissue elasticity: measurements by atomic force microscopy and its influence on cell differentiation. Methods in cell biology. 2007; 83:521-545. [PubMed: 17613323]

6. Discher DE, Janmey P, Wang YL. Tissue cells feel and respond to the stiffness of their substrate. Science. 2005; 310:1139-1143. [PubMed: 16293750]

7. Khorasani H, et al. A Quantitative Approach to Scar Analysis. American Journal of Pathology. 2011; 178:621-628. [PubMed: 21281794]

8. Martin P. Wound healing--aiming for perfect skin regeneration. Science. 1997; 276:75-81. [PubMed: 9082989]

9. Bakay M, Zhao P, Chen J, Hoffman EP. A web-accessible complete transcriptome of normal human and DMD muscle. Neuromuscular disorders : NMD. 2002; 12(Suppl 1):S125-S141. [PubMed: 12206807]

10. Hinz B. Formation and function of the myofibroblast during tissue repair. The Journal of investigative dermatology. 2007; 127:526-537. [PubMed: 17299435]

11. Willems IEMG, Havenith MG, Demey JGR, Daemen MJAP. The Alpha-Smooth Muscle ActinPositive Cells in Healing Human Myocardial Scars. American Journal of Pathology. 1994; 145:868-875. [PubMed: 7943177]

12. Swift J, et al. Nuclear lamin-A scales with tissue stiffness and enhances matrix-directed differentiation. Science. 2013; 341:1240104. [PubMed: 23990565]

13. Kasahara H, Bartunkova S, Schinke M, Tanaka M, Izumo S. Cardiac and extracardiac expression of Csx/Nkx2.5 homeodomain protein. Circulation research. 1998; 82:936-946. [PubMed: 9598591]

14. Pittenger MF, et al. Multilineage potential of adult human mesenchymal stem cells. Science. 1999; 284:143-147. [PubMed: 10102814]

15. Hare JM, et al. A randomized, double-blind, placebo-controlled, dose-escalation study of intravenous adult human mesenchymal stem cells (prochymal) after acute myocardial infarction. Journal of the American College of Cardiology. 2009; 54:2277-2286. [PubMed: 19958962]

16. Engler AJ, Sen S, Sweeney HL, Discher DE. Matrix elasticity directs stem cell lineage specification. Cell. 2006; 126:677-689. [PubMed: 16923388]

17. Winer JP, Janmey PA, McCormick ME, Funaki M. Bone Marrow-Derived Human Mesenchymal Stem Cells Become Quiescent on Soft Substrates but Remain Responsive to Chemical or Mechanical Stimuli. Tissue Eng Pt A. 2009; 15:147-154.

18. Larson BL, Ylostalo J, Prockop DJ. Human multipotent stromal cells undergo sharp transition from division to development in culture. Stem Cells. 2008; 26:193-201. [PubMed: 17916801]

19. Barberi T, Willis LM, Socci ND, Studer L. Derivation of multipotent mesenchymal precursors from human embryonic stem cells. Plos Med. 2005; 2:554-560.

20. Wong S, Guo WH, Hoffecker I, Wang YL. Preparation of a micropatterned rigid-soft composite substrate for probing cellular rigidity sensing. Methods in cell biology. 2014; 121:3-15. [PubMed: 24560499]

21. Benoit DS, Schwartz MP, Durney AR, Anseth KS. Small functional groups for controlled differentiation of hydrogel-encapsulated human mesenchymal stem cells. Nature materials. 2008; 7:816-823. [PubMed: 18724374]

22. Huebsch N, et al. Harnessing traction-mediated manipulation of the cell/matrix interface to control stem-cell fate. Nature materials. 2010; 9:518-526. [PubMed: 20418863]

23. Khetan $\mathrm{S}$, et al. Degradation-mediated cellular traction directs stem cell fate in covalently crosslinked three-dimensional hydrogels. Nature materials. 2013; 12:458-465. [PubMed: 23524375]

24. Trappmann B, et al. Extracellular-matrix tethering regulates stem-cell fate. Nature materials. 2012; 11:642-649. [PubMed: 22635042]

25. McNamara LE, et al. Nanotopographical control of stem cell differentiation. Journal of tissue engineering. 2010; 2010:120623. [PubMed: 21350640] 
26. Corr DT, Gallant-Behm CL, Shrive NG, Hart DA. Biomechanical behavior of scar tissue and uninjured skin in a porcine model. Wound Repair Regen. 2009; 17:250-259. [PubMed: 19320894]

27. Achterberg VF, et al. The nano-scale mechanical properties of the extracellular matrix regulate dermal fibroblast function. The Journal of investigative dermatology. 2014; 134:1862-1872. [PubMed: 24670384]

28. MacQueen L, Sun Y, Simmons CA. Mesenchymal stem cell mechanobiology and emerging experimental platforms. Journal of the Royal Society, Interface / the Royal Society. 2013; 10:20130179.

29. Dembo M, Wang YL. Stresses at the cell-to-substrate interface during locomotion of fibroblasts. Biophysical journal. 1999; 76:2307-2316. [PubMed: 10096925]

30. Storm C, Pastore JJ, MacKintosh FC, Lubensky TC, Janmey PA. Nonlinear elasticity in biological gels. Nature. 2005; 435:191-194. [PubMed: 15889088]

31. Vader D, Kabla A, Weitz D, Mahadevan L. Strain-Induced Alignment in Collagen Gels. PloS one. 2009; 4

32. Buxboim A, Rajagopal K, Brown AE, Discher DE. How deeply cells feel: methods for thin gels. Journal of physics. Condensed matter : an Institute of Physics journal. 2010; 22:194116. [PubMed: 20454525]

33. Engler A, et al. Substrate compliance versus ligand density in cell on gel responses. Biophysical journal. 2004; 86:617-628. [PubMed: 14695306]

34. Mann CJ, et al. Aberrant repair and fibrosis development in skeletal muscle. Skeletal muscle. 2011; 1:21. [PubMed: 21798099]

35. Raab M, et al. Crawling from soft to stiff matrix polarizes the cytoskeleton and phosphoregulates myosin-II heavy chain. The Journal of cell biology. 2012; 199:669-683. [PubMed: 23128239]

36. Zemel A, Rehfeldt F, Brown AEX, Discher DE, Safran SA. Cell shape, spreading symmetry, and the polarization of stress-fibers in cells. J Phys-Condens Mat. 2010; 22

37. Shin JW, et al. Contractile Forces Sustain and Polarize Hematopoiesis from Stem and Progenitor Cells. Cell stem cell. 2014; 14:81-93. [PubMed: 24268694]

38. Schmiedel JM, et al. MicroRNA control of protein expression noise. Science. 2015; 348:128-132. [PubMed: 25838385]

39. Rinkevich Y, et al. Skin fibrosis. Identification and isolation of a dermal lineage with intrinsic fibrogenic potential. Science. 2015; 348:aaa2151. [PubMed: 25883361]

40. Miralles F, Posern G, Zaromytidou AI, Treisman R. Actin dynamics control SRF activity by regulation of its coactivator MAL. Cell. 2003; 113:329-342. [PubMed: 12732141]

41. Dupont S, et al. Role of YAP/TAZ in mechanotransduction. Nature. 2011; 474:179-U212. [PubMed: 21654799]

42. Sullivan KE, Quinn KP, Tang KM, Georgakoudi I, Black LD. Extracellular matrix remodeling following myocardial infarction influences the therapeutic potential of mesenchymal stem cells. Stem Cell Res Ther. 2014; 5

43. Couzin-Frankel J. The elusive heart fix. Science. 2014; 345:252-257. [PubMed: 25035469]

44. Kasahara H, Izumo S. Identification of the in vivo casein kinase II phosphorylation site within the homeodomain of the cardiac tissue-specifying homeobox gene product Csx/Nkx2.5. Mol Cell Biol. 1999; 19:526-536. [PubMed: 9858576]

45. Ryan T, et al. Myosin Phosphatase Modulates the Cardiac Cell Fate by Regulating the Subcellular Localization of Nkx2.5 in a Wnt/Rho-Associated Protein Kinase-Dependent Pathway. Circ Res. 2013; 112:257. [PubMed: 23168335]

46. Yang C, Tibbitt MW, Basta L, Anseth KS. Mechanical memory and dosing influence stem cell fate. Nature materials. 2014

47. Costa MW, et al. Complex SUMO-1 Regulation of Cardiac Transcription Factor Nkx2-5. PloS one. 2011; 6

48. Breitbach $\mathrm{M}$, et al. Potential risks of bone marrow cell transplantation into infarcted hearts. Blood. 2007; 110:1362-1369. [PubMed: 17483296] 
49. Dingal PC, Discher DE. Systems mechanobiology: tension-inhibited protein turnover is sufficient to physically control gene circuits. Biophysical journal. 2014; 107:2734-2743. [PubMed: 25468352]

50. Rehfeldt F, et al. Hyaluronic acid matrices show matrix stiffness in 2D and 3D dictates cytoskeletal order and myosin-II phosphorylation within stem cells. Integrative biology : quantitative biosciences from nano to macro. 2012; 4:422-430. [PubMed: 22344328]

51. Addis RC, et al. Optimization of direct fibroblast reprogramming to cardiomyocytes using calcium activity as a functional measure of success. J Mol Cell Cardiol. 2013; 60:97-106. [PubMed: 23591016]

52. Lo CM, et al. Nonmuscle myosin IIb is involved in the guidance of fibroblast migration. Molecular biology of the cell. 2004; 15:982-989. [PubMed: 14699073]

53. Domke J, Radmacher M. Measuring the elastic properties of thin polymer films with the atomic force microscope. Langmuir. 1998; 14:3320-3325. 

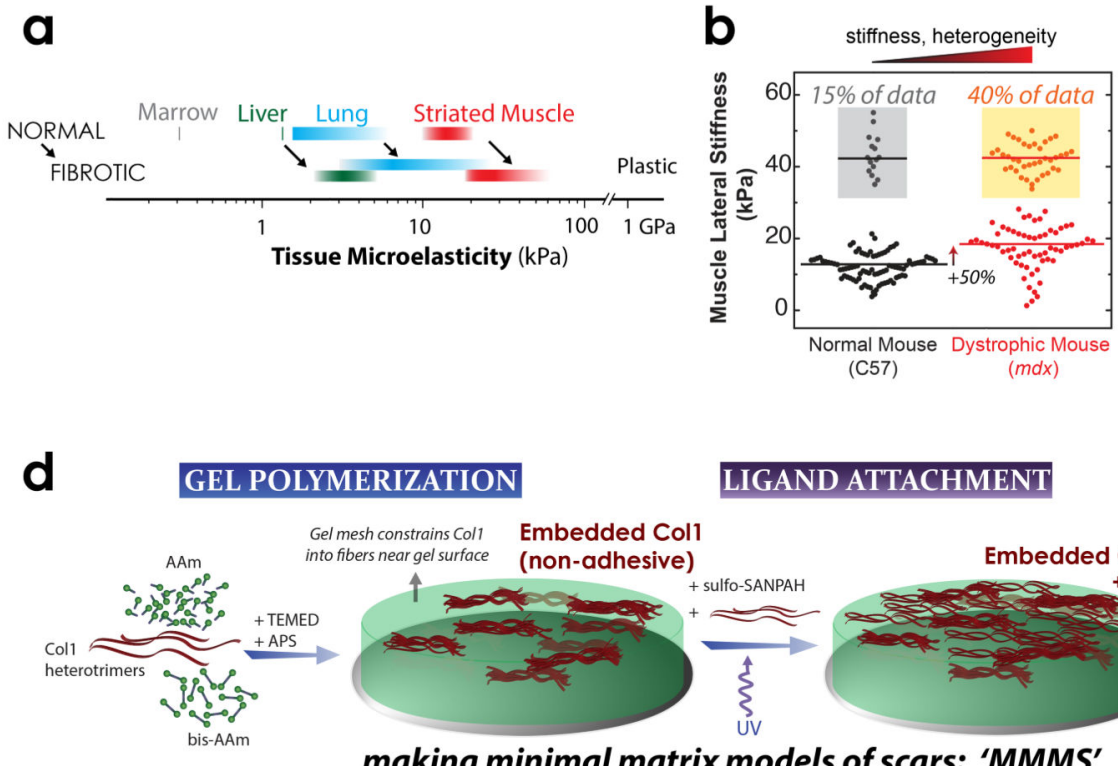

Gel mesh constrains Col1 Embedded Coll

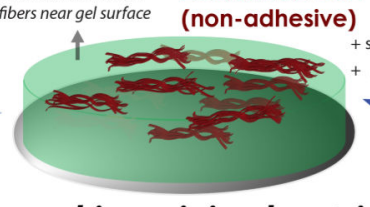

making minimal matrix models of scars: 'MMMS'
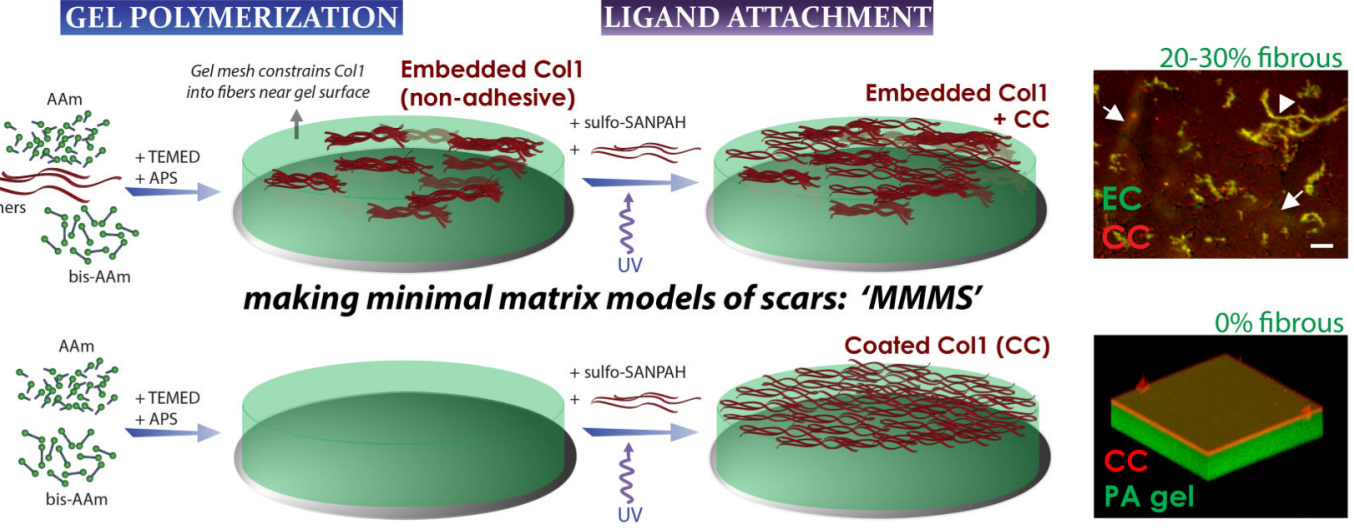

MMMS:

Heterogeneous Substrate, Homogeneous Ligand

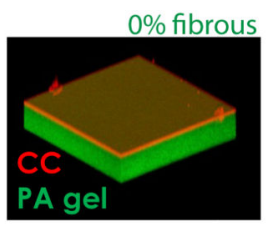

Conventional Substrate: Homogeneous

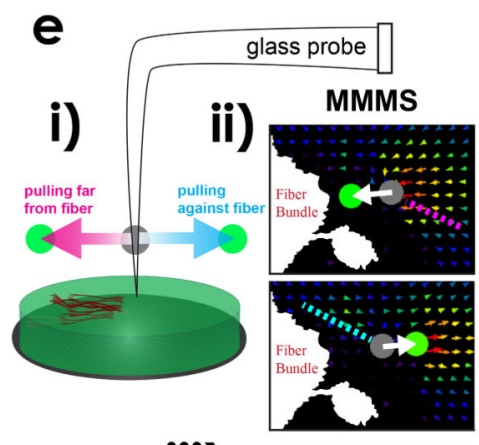

iii)
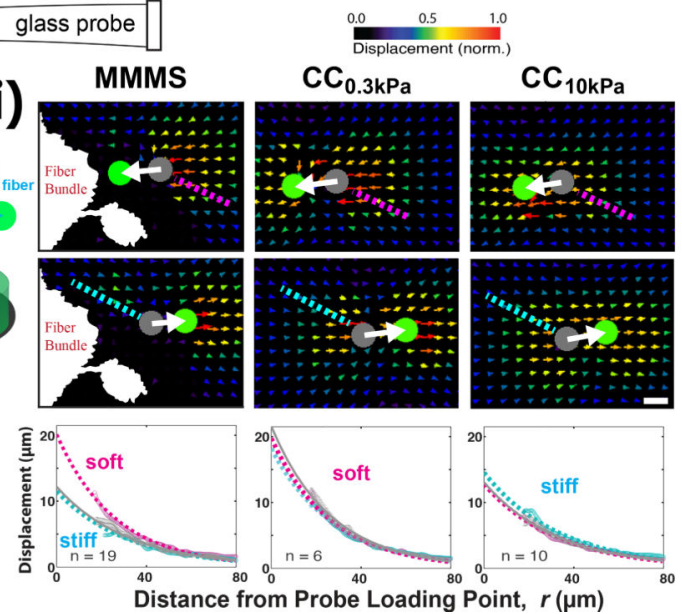

f

Figure 1. A minimal matrix model of scars, MMMS

a, Fibrosis-associated stiffening and heterogeneity is consistently seen across tissues with abundant collagen such as liver ${ }^{2}$, lung ${ }^{3}$ and striated muscle ${ }^{4,5}$. b, $m d x$ mouse muscle tissue is stiffer and more heterogeneous than normal mouse (C57) . c, At the transcript level, structural genes such as COL1 (median of COL1A1 and COL1A2), LMNA, ACTA2 and $C A L D 1$ upregulate in fibrotic muscle tissue ${ }^{9}$; parallel increases are also seen in long-term (vs short-term) cultures of $\mathrm{MSC}^{18}$ and embryonic stem cell (ESC)-derived MSC (vs ESC) ${ }^{19}$. Transcription factors relevant in mechanotransduction pathways either scale with $(R A R G)$, do not scale (SRF), or scale against (NKX2-5) COL1 levels. $m d x=$ mouse muscular 
dystrophy model; Norm = normal human muscle; BMD = Becker's muscular dystrophy; DMD = Duchenne muscular dystrophy. $\mathbf{d}$, (Top panel) A minimal matrix model of scars (MMMS) is created by incorporating $400 \mu \mathrm{g} \mathrm{mL}{ }^{-1}$ of collagen-1 during free-radical polymerization of a polyacrylamide (PA). (Top, right) Fiber bundles of embedded collagen (EC, green) in coated-collagen (CC, red) $0.3 \mathrm{kPa}$ PA gel. Most EC bundles localize near the surface (arrowhead), while some are more deeply embedded (arrows), creating a heterogeneous thin film. Scale bar, $100 \mu \mathrm{m}$. (Bottom panel) Conventional collagen-I matrix attachment on PA gel. (Bottom, right) PA gel (green) with CC (red) reconstructed from confocal image stacks ( $365 \mu \mathrm{m} \times 365 \mu \mathrm{m}$ surface, $\sim 80 \mu \mathrm{m}$ height). e, i) Lateral pullings in MMMS gels indicate ii) heterogeneous Boussinesq-like displacement profiles by tracking movement of embedded microbeads in the gels. iii) Pulling against a fiber bundle in MMMS gel shows smaller bead displacements (left, cyan and grey) similar to a stiffer 10kPa gel (right). Pulling far from a bundle (left, magenta) has a displacement profile similar to a $0.3 \mathrm{kPa}$ gel (center). Grey curves are averaged profiles from $n$ pullings. Scale bar, $10 \mu \mathrm{m}$. f, Schematic: Fiber bundles in the same focal plane as beads settled on the gel surface. Immunolabeled fiber bundles (top, left) stain positive for Sirius Red (top, middle). Higher magnification of Sirius Red-positive EC fibers (top, right). CC gels (bottom) have no significant Sirius Red staining, as revealed by line intensity scans (inset). g, Sirius Red staining of a thin section of fibrotic liver. Scale bars, $100 \mu \mathrm{m}$. 


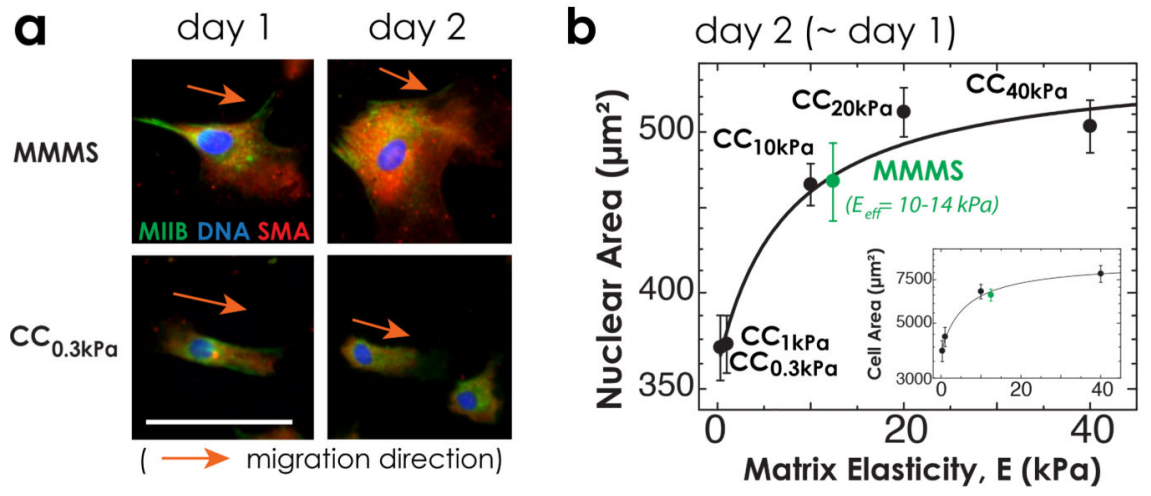

d
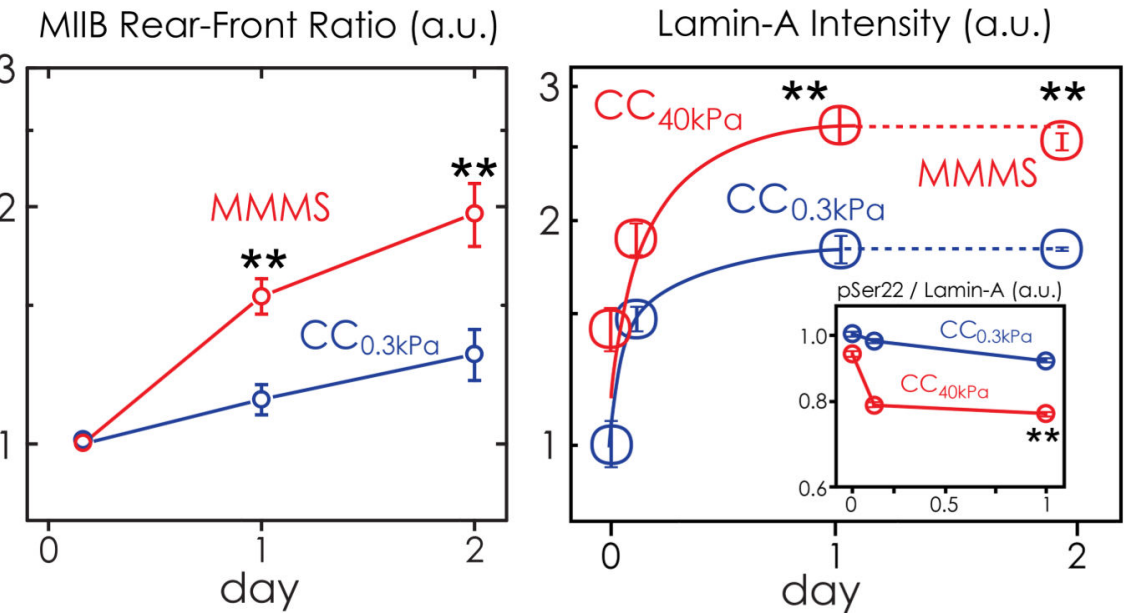

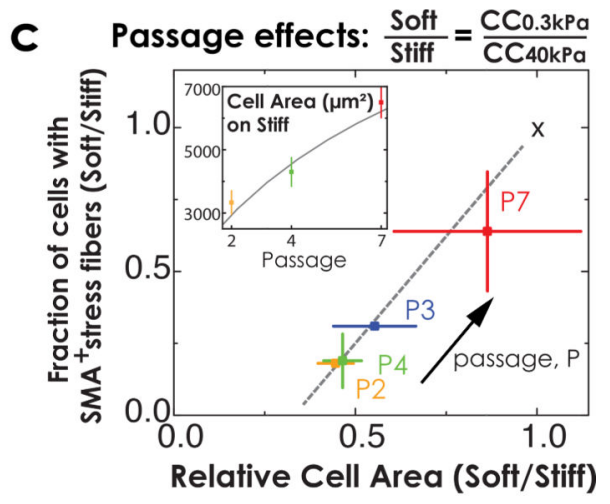

Figure 2. Mechanosensitive proteins respond at different time-scales to matrix stiffness a, Immunofluorescence images of myosin-IIB (MIIB, green) and smooth muscle actin (SMA, red) at 1 and 2 days after seeding MSCs on MMMS (top) and $\mathrm{CC}_{0.3 \mathrm{kPa}}$ gels (bottom). DNA, blue. Scale bar, $100 \mu \mathrm{m}$. b, Cell morphological effects of matrix elasticity, $E$, are fitted with a hyperbolic equation: $A+B \cdot E /\left(E_{m}+E\right)$ using a characteristic elasticity constant, $E_{m}=9.2 \mathrm{kPa}$ (ref. $\left.{ }^{50}\right)$. Matrix elasticity-dependent cell $\left(A=3797 \mu \mathrm{m}^{2}, B=5064\right)$ and nuclear (inset; $A=360 \mu \mathrm{m}^{2}, B=191$ ) spread area are quantified (mean \pm s.e.m.) from nonmuscle myosin-IIA immunofluorescence staining of MSCs cultured for 1-2 days on gels ( $n=3$ independent experiments). c, Passage (P) effects on MSC area and cell fraction with SMA-positive stress fibers on soft $\left(\mathrm{CC}_{0.3 \mathrm{kPa}}\right)$ versus stiff $\left(\mathrm{CC}_{40 \mathrm{kPa}}\right)$ matrix (from multiple donors; $n=2$ per passage). At very high passage, cell becomes insensitive to matrix stiffness $(\times)$. Inset: Cell area on stiff matrix of single-donor MSCs tracked over passage (fit: $880+$ $12400 P$ / $(9+\mathrm{P})$; grey curve), mean \pm s.e.m. d, Expression kinetics of mechanosensitive protein in MSCs on gels. left, MIIB rearward polarization of $\mathrm{MSCs}$ on $\mathrm{CC}_{0.3 \mathrm{kPa}}$ (blue) or MMMS (red) occured within a day of culture $(n=3)$; middle, Expression of lamin-A (and its phosphorylation at Serine-22; inset) was even faster $(<12 \mathrm{~h})$, and higher (with lower phosphorylation levels) on stiff $\mathrm{CC}_{40 \mathrm{kPa}}$ and MMMS (red) than on $\mathrm{CC}_{0.3 \mathrm{kPa}}$ (blue) gel ( $n=$ $3)$; right, SMA levels did not increase until 2 days of culture on stiff gels $(n \geq 2)$. (Inset: 
Quantitative immunofluorescence of SMA ( $A=1, B=1.17$ ) vs $E$ after 2 days in culture; $n=$ 4). Mean \pm s.e.m. ${ }^{* *} p<0.01$, when compared to $\mathrm{CC}_{0.3 \mathrm{kPa}}$ gels. Goodness of fits, $\mathrm{R}^{2} \geq 0.95$. 

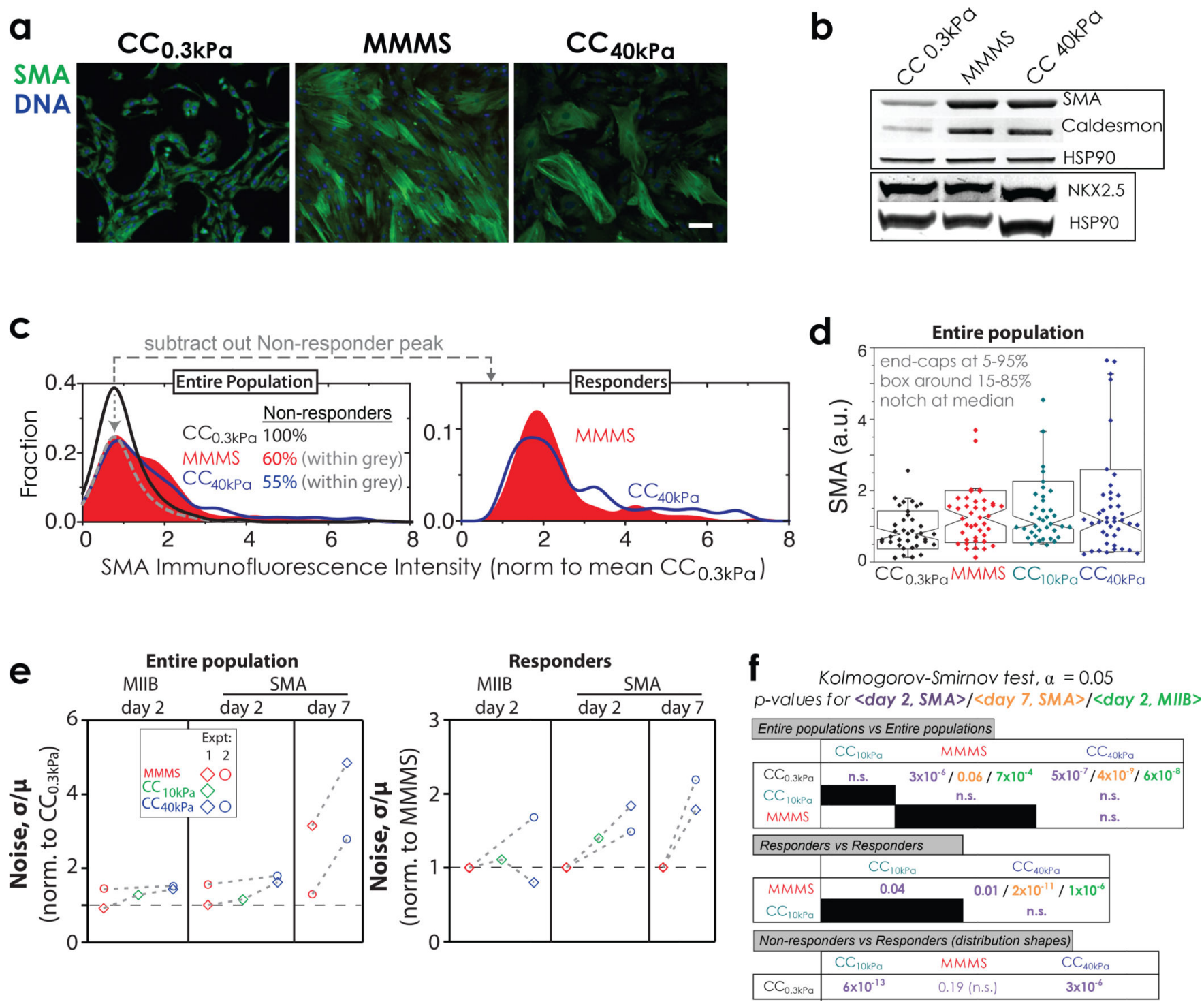

Figure 3. Cell-to-cell variation is lower on MMMS than on homogeneously stiff substrates a, SMA expression (green) on MMMS is less heterogeneous than on $\mathrm{CC}_{40 \mathrm{kPa}}$. SMA incorporated into stress fibers over 7 days on both MMMS and $\mathrm{CC}_{40 \mathrm{kPa}}$, but did not on $\mathrm{CC}_{0.3 \mathrm{kPa}}$. DNA, blue. Scale bar, $100 \mu \mathrm{m}$. b, Immunoblot shows overall increase in SMA and Caldesmon in MSCs cultured for 7 days on MMMS and $\mathrm{CC}_{40 \mathrm{kPa}}$ relative to $\mathrm{CC}_{0.3 \mathrm{kPa}}$. Total NKX2.5 levels did not change in response to matrix stiffness. HSP90 as loading control. c, Left histogram shows SMA intensity per cell relative to mean level of $\mathrm{CC}_{0.3 \mathrm{kPa}}$ in 2 -day cultures $(n=3)$ (also per Fig. 2d). MSCs on $\mathrm{CC}_{0.3 \mathrm{kPa}}$ are Non-responders to matrix stiffness (black curve). Grey curve is the $\mathrm{CC}_{0.3 \mathrm{kPa}}$ peak re-scaled to the peak heights of MMMS and $\mathrm{CC}_{40 \mathrm{kPa}}$. The grey curve envelopes $60 \%$ of the entire population of cells on MMMS and $55 \%$ on $\mathrm{CC}_{40 \mathrm{kPa}}$. These same cells show both low SMA and low MIIB (Supplementary Fig. S9). Right histogram is obtained by subtracting out Non-responder subpopulations to obtain Responder populations on MMMS and on stiff, homogeneous gels (see Methods). d-f, Homogeneous gels $\mathrm{CC}_{10 \mathrm{kPa}}$ and $\mathrm{CC}_{40 \mathrm{kPa}}$ elicit broader distributions than MMMS: d, Box- 
and-whisker scatterplots of SMA levels at single-cell level: whisker end-caps at 5-95\%, box encompassing 15-85\% $(\sim 1.5 \sigma)$, and notch at the median $(n=2)$. e, Single-cell expression noise quantified as the ratio of standard deviation and mean, $\sigma / \mu(n=2)$. $\mathbf{f}, p$-values for each two-sample Kolmogorov-Smirnov test, which tests for higher modes of the distributions, at $a=0.05$. 

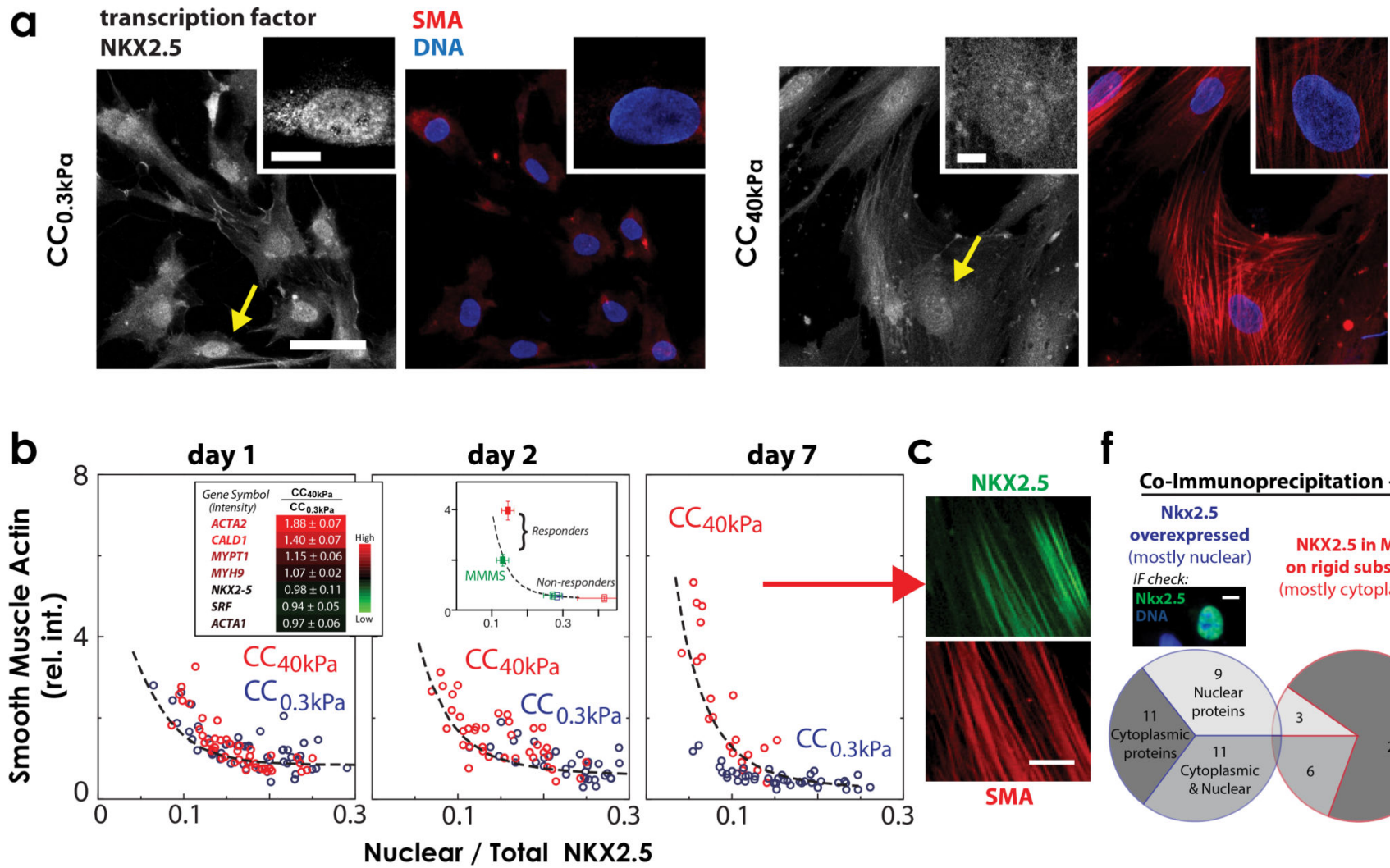

f

Nuclear / Total NKX2.5
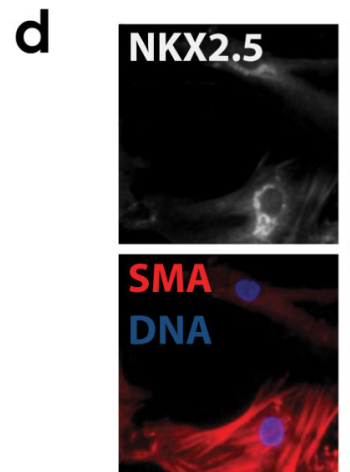

$\mathrm{Oh}$
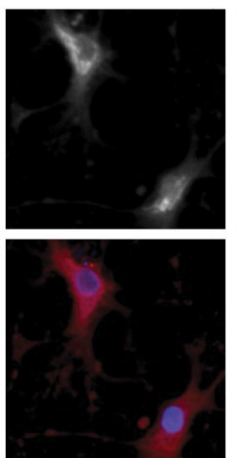

$4 \mathrm{~h}$
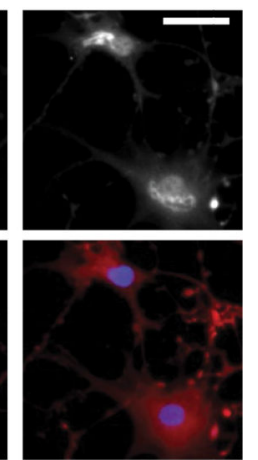

$1 \mathrm{~d}$

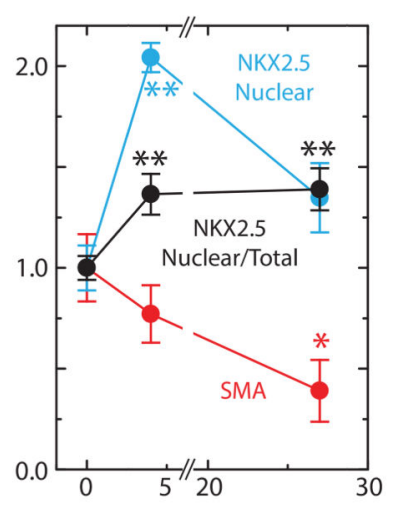

Blebbistatin Treatment Time (h) e

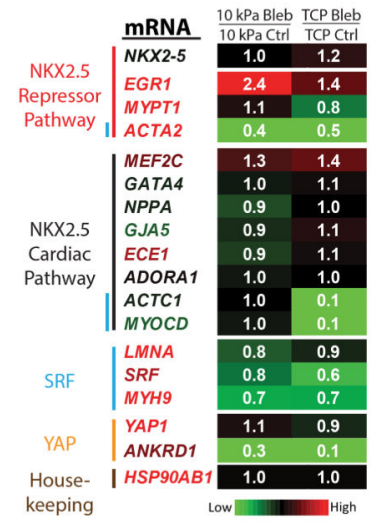

Figure 4. Matrix stiffness and cell tension modulates NKX2.5 expression and delayed shuttling a, Immunofluorescence staining of NKX2.5 (left) and SMA (right, red) in MSCs on soft $\left(\mathrm{CC}_{0.3 \mathrm{kPa}}\right)$ and stiff $\left(\mathrm{CC}_{40 \mathrm{kPa}}\right)$ gels for 7 days. Scale bars, $50 \mu \mathrm{m}$. Insets: Confocal image slices of representative cell nuclei (arrows); scale bars, $10 \mu \mathrm{m}$. b, MSCs cultured for 1 day (left), 2 days (middle) or 7 days (right) on soft (blue) or stiff (red) gels differed in their dynamic range of SMA expression $(n=4)$. NKX2.5 depletion from the nucleus took several days to obtain $\sim 8$-fold increase of SMA. SMA-NKX2.5 response (dashed lines) obey a typical cooperative inhibition model: $y=A\left[1-x^{2.7} /\left(0.06^{2.7}+x^{2.7}\right)\right]+B$; where $x, y, A+B$, $B$ and 2.7 are nuclear/total NKX2.5, SMA, $y$-intercept, $x$-intercept and cooperativity exponent, respectively. Fit values: (day $\#: A, B, R^{2}$ of fit $)=[($ day $1: 3.7,0.7,0.80)$, (day 2 : 
5.7, 0.6, 0.86), (day 7: 7.0, 0, 0.96)]. Increasing $A$ from day 1 to day 7 might reflect a paucity of data at low values of $x$. Left inset: Microarray profiling of MSCs cultured for $36 \mathrm{~h}$ on stiff $\left(\mathrm{CC}_{40 \mathrm{kPa}}\right)$ gels revealed ACTA2 upregulation, but not $N K X 2-5, S R F$ and a downstream target of SRF, MYH9, when compared to soft $\left(\mathrm{CC}_{0.3 \mathrm{kPa}}\right)$ gels $(n=5$; mean \pm s.d.). Right inset: NKX2.5-SMA variations of MSCs on MMMS at day-2 (independent experiment, see also Supplementary Fig. S9). Differences in Responder populations on MMMS and the CC40kPa are statistically significant $(p<0.05)$. $\mathbf{c}, \mathrm{NKX} 2.5$ is found on stress fibers in MSCs cultured on a rigid substrate. Scale bar, $20 \mu \mathrm{m}$. d, Kinetics of NKX2.5 expression and localization in MSCs treated with blebbistatin for 4-27 hrs revealed loss of SMA (red) and nuclear enrichment of NKX2.5 (cyan, black). DNA, blue. Mean \pm s.e.m ( $n=$ 2). Scale bars, $50 \mu \mathrm{m}$. e, Transcriptomic profiling of MSCs treated with blebbistatin on $\mathrm{CC}_{10 \mathrm{kPa}}$ gels or rigid plastic (TCP) for $24 \mathrm{hrs}$ revealed decreased $S R F$ and SRF-target genes and downstream targets of NKX2.5 related to cardiac specification were downregulated and/or lowly-expressed $(n=2)$. f, Mass Spectrometry (MS) analyses revealed proteins that co-immunoprecipitated (CoIP) with cytoplasm-enriched NKX2.5 (in lysates of MSCs on rigid substrate), or with overexpressed nuclear Nkx2.5 (in A549 cell lysates) but not with a nonspecific antibody control (IgG). For consistency, nuclear localization of overexpressed Nkx2.5 was confirmed with mutagenesis and immunofluorescence (IF) with the same antiNkx2.5 antibody used in CoIP-MS (see also Supplementary Fig. S10). Scale bar, $10 \mu \mathrm{m}$. 


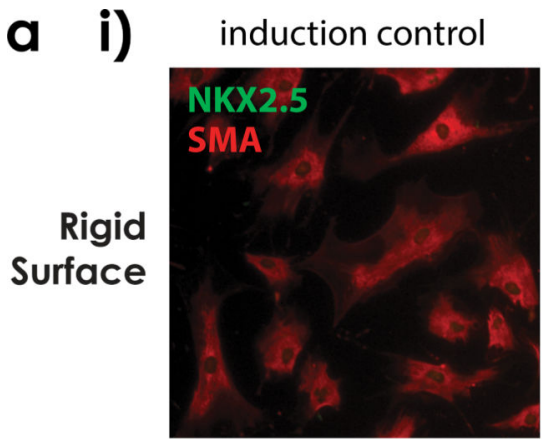

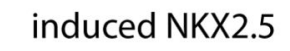

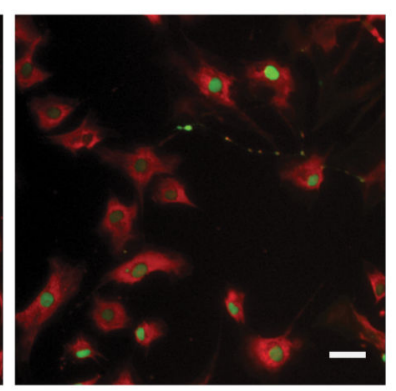

ii)

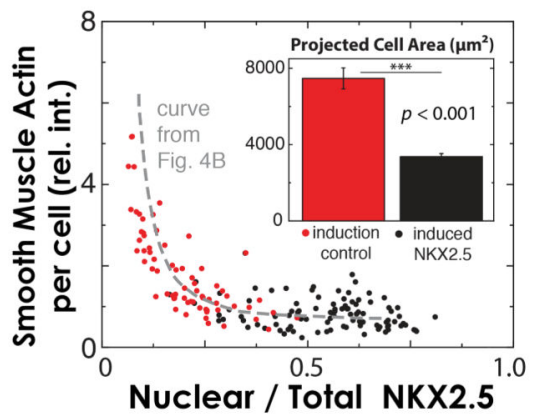

b NKX2.5 with mutations in its Nuclear Localization Signal (NLS)
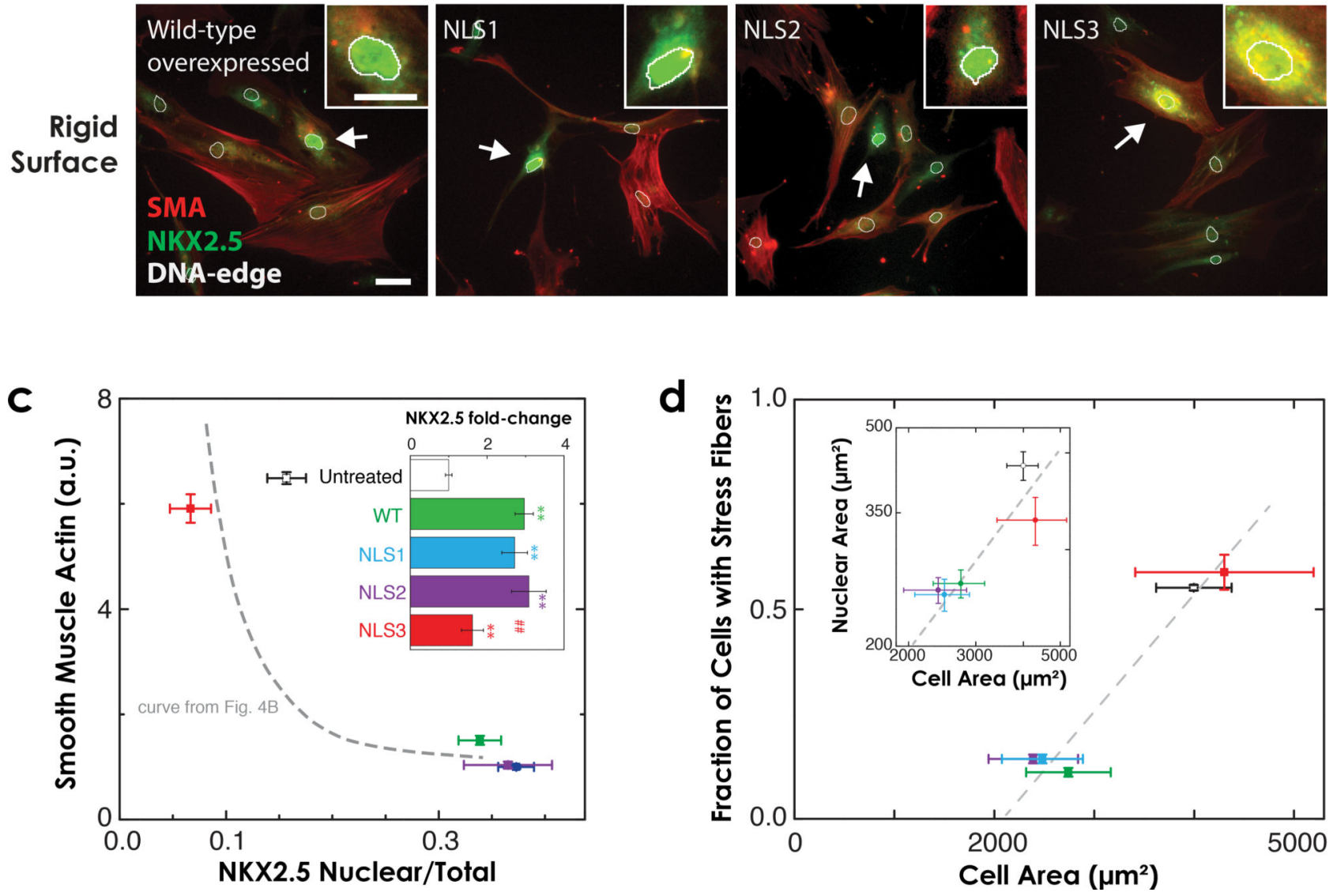

Figure 5. NKX2.5 overexpression resets the mechanical memory of the rigid-substrate phenotype a, i) A doxycycline (DOX)-inducible NKX2.5 lentiviral vector (in combination with the reverse tetracycline transactivator, rtTA, vector) ${ }^{51}$ was introduced into late-passage MSCs (P6). Doxycycline treatment $(2 \mu \mathrm{g} / \mathrm{mL})$ activated transgene expression of NKX2.5 (right) versus rtTA-only control (left) and observed ii) reduced SMA levels and projected cell areas (inset) after 2 days, as if on soft matrix ( $n=2$; mean \pm s.e.m.). An inhibition model from Fig. $4 \mathrm{~b}$ is fitted here (grey curve): $y=20\left[1-x^{2.7} /\left(0.06^{2.7}+x^{2.7}\right)\right]+0.7$. b, Overexpression of wild-type (WT) Nkx2.5 or its nuclear localization sequence (NLS) mutants (green) in 
MSCs (white arrows) cultured on rigid plastic for 2 days post-transfection.. Insets: Magnified nuclei of Nkx2.5-overexpressing MSCs. DNA-edge (white) is superimposed. Scale bars, $50 \mu \mathrm{m}$. c, Inverse relationship between SMA and Nuclear/Total ratio of Nkx2.5 with ectopic expression of Nkx2.5 NLS mutants. SMA expression is abrogated with overexpressed, nuclear-localized Nkx2.5 (WT, NLS1, NLS2) but not with cytoplasmic NLS3. Curve fit (grey line): $y=20\left[1-x^{2.7} /\left(0.06^{2.7}+x^{2.7}\right)\right]+1$; Inset: Fold-changes in Nkx2.5 intensity upon overexpression. $\mathbf{d}$, Cell fraction with stress fibers decrease with ectopic addition of nuclear-localized $\mathrm{Nkx} 2.5$, with linear fit showing intercept analogous to Fig. 2c. Inset: concomitant reduction of nuclear area with cell area, as expected from Fig. 2b. Mean \pm s.e.m $(n=2)$. $* * p<0.01$ when compared to untreated control (UT). \#\#p $<0.01$ when compared to WT, NLS1, and NLS2. 
a
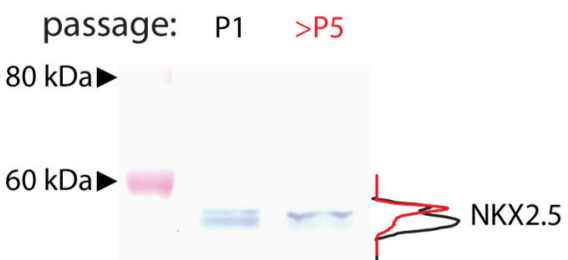

$50 \mathrm{kDa}$

$40 \mathrm{kDa}$
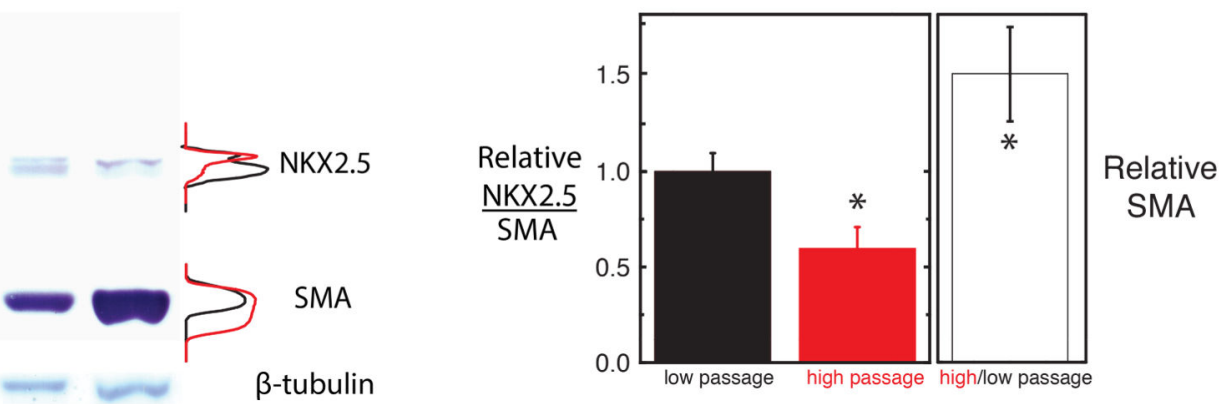

b

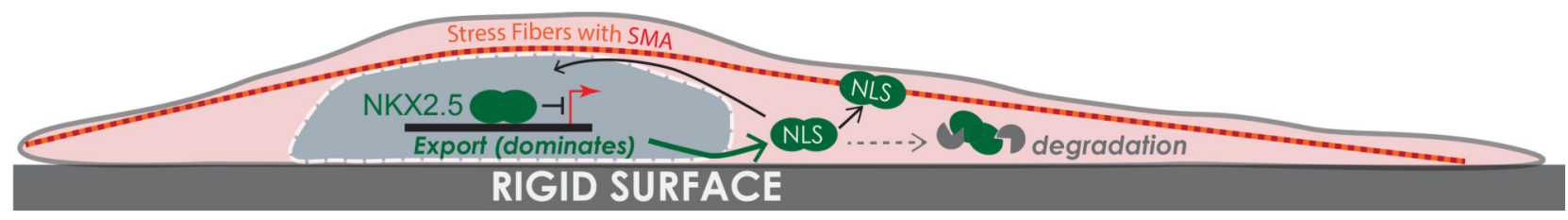

Figure 6. NKX2.5 degrades in long-term stiff-matrix cultures to set a mechanical memory

a, Left, Immunoblot revealed that long-term culture (high passage, >P5) of MSCs on tissue culture plastic led to decreased levels of NKX2.5, while SMA levels are increased. $\beta$-tubulin as loading control. Right, Quantification of NKX2.5/SMA and SMA (normalized to $\beta$ tubulin) levels with passage $(n=4)$. Mean \pm s.e.m. $* p<0.05$ compared to low passage MSCs. b, Culturing MSCs on rigid substrates leads to nuclear export of NKX2.5, which can interact with stress fibers and/or degrade (see Supplementary Discussion). 